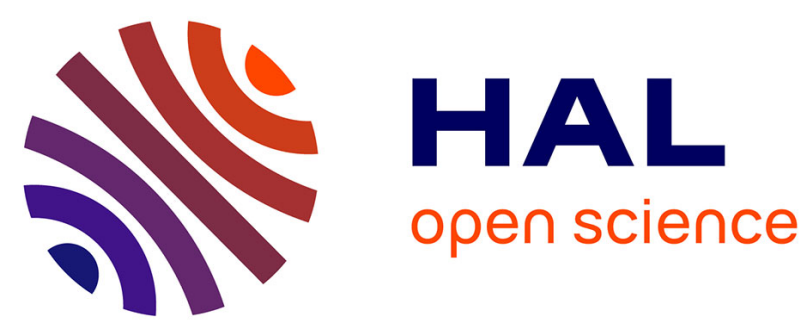

\title{
Transmission gaps and Fano resonances in an acoustic waveguide: analytical model
}

El Houssaine El Boudouti, Tarik Mrabti, Housni Al-Wahsh, Bahram

Djafari-Rouhani, Abdellatif Akjouj, L. Dobrzynski

\section{To cite this version:}

El Houssaine El Boudouti, Tarik Mrabti, Housni Al-Wahsh, Bahram Djafari-Rouhani, Abdellatif Akjouj, et al.. Transmission gaps and Fano resonances in an acoustic waveguide: analytical model. Journal of Physics: Condensed Matter, 2008, 20, pp.255212-1-10. 10.1088/0953-8984/20/25/255212 . hal-00357776

\section{HAL Id: hal-00357776 https://hal.science/hal-00357776}

Submitted on 31 Aug 2021

HAL is a multi-disciplinary open access archive for the deposit and dissemination of scientific research documents, whether they are published or not. The documents may come from teaching and research institutions in France or abroad, or from public or private research centers.
L'archive ouverte pluridisciplinaire HAL, est destinée au dépôt et à la diffusion de documents scientifiques de niveau recherche, publiés ou non, émanant des établissements d'enseignement et de recherche français ou étrangers, des laboratoires publics ou privés. 


\title{
Transmission gaps and Fano resonances in an acoustic waveguide: analytical model
}

\author{
E H El Boudouti ${ }^{1}$, T Mrabti ${ }^{1}$, H Al-Wahsh ${ }^{2}$, B Djafari-Rouhani ${ }^{3}$, \\ A Akjouj ${ }^{3,4}$ and L Dobrzynski ${ }^{3}$ \\ ${ }^{1}$ Laboratoire de Dynamique et d'optique des matériaux, Département de Physique, \\ Faculté des Sciences, Université Mohamed I, Oujda, Morocco \\ ${ }^{2}$ Faculty of Engineering, Benha University, 11241 Cairo, Egypt \\ ${ }^{3}$ Institut d'Electronique, de Microélectronique et de Nanotechnologie (IEMN), UMR CNRS \\ 8520, UFR de Physique, Université des Sciences et Technologies de Lille, 59655 Villeneuve \\ d'Ascq Cédex, France \\ E-mail: Abdellatif.Akjouj@univ-lille1.fr
}

\begin{abstract}
A simple acoustic device consisting of two dangling side resonators grafted at two sites on a slender tube is designed possibly to obtain transmission stop bands (where the propagation of longitudinal acoustic waves is forbidden). In contrast to all known systems of this kind, a spectral transmission gap of nonzero width occurs here even with this simple structure. This is obtained by combining appropriately the zeros of transmission of the side resonators. Sharp resonant states inside the gaps can be achieved without introducing any defects in the structure. This results from an internal resonance of the structure when such a resonance is situated in the vicinity of a zero of transmission or placed between two zeros of transmission, the so-called Fano resonances. A general analytical expression for the transmission coefficient is given for various systems of this kind within the framework of the Green's function method. The amplitude and the phase of the transmission are discussed as a function of frequency and it is shown that the width of the stop bands is very sensitive to the number of side resonators. These results should have important consequences for the suppression of low-frequency noise and for designing filters.
\end{abstract}

\section{Introduction}

Low dimensional structures which have a regular distribution of scattering centers have been seen to possess a distinct and interesting array of acoustical properties, perhaps most strikingly frequency band gaps within which acoustic waves cannot propagate through the structure-a so-called phononic band gap. In recent years, such phononic crystals have been the subject of intense theoretical [1] and experimental [2] investigations. Two-and three-dimensional composite systems constituted by periodic inclusions of a given material in a host matrix can exhibit an absolute acoustic band gap where the propagation of sound waves and ultrasonic vibrations is inhibited in any direction of space [3]. These acoustic band gap materials can have many practical applications such as elastic/acoustic filters [4]. Studies of lower dimensional

\footnotetext{
4 Author to whom any correspondence should be addressed.
}

systems such as 1D periodic layered media [5, 6] and periodic waveguide systems with different geometries [7-13] are conducted as analogs of $2 \mathrm{D}$ and $3 \mathrm{D}$ systems and for applications in their own right. These structures are attractive since their production is more feasible at any wavelength scale and they require only simple analytical and numerical calculations.

In a previous publication [9], some of the authors reported that the acoustic transmission spectrum of 1D comb structures exhibits large gaps. These structures, called star wave guides, are composed of $N^{\prime}$ dangling side branches (DSBs) periodically grafted at each of the $N$ equidistant sites on slender tubes. The gaps originate from the periodicity of the system determined by the distance between two neighboring sites and from the eigenfrequencies of the DSB, which play the role of resonators. These theoretical results are confirmed by experiments using an impulse response technique in the 
interval from 650 to $1100 \mathrm{~Hz}$ [12]. Unlike other 1D (e.g. Bragg lattices), 2D or 3D phononic crystals, in which the contrast between the constituents is a critical parameter for the stop band existence, this star waveguide exhibits relatively large forbidden bands even if the backbone and the resonators are made of the same material [9].

On the other hand, we have proposed [13] an asymmetric serial loop structure made of asymmetric slender tube loops pasted together with slender tubes of finite length; the loops play the role of resonators. Such a structure exhibits new features, in comparison with the star waveguide [9]. In the case of symmetric loops, the system becomes equivalent to a diameter modulated slender tube [8]. Recently, we have also investigated theoretically the propagation and localization of acoustic waves in quasi-periodic [14] slender tubes and these results have been confirmed experimentally [15]. In addition, we have shown the existence of transmission zeros in the case of asymmetric loop structure [14]. These transmission zeros may lead to a phase drop of $\pi$ and therefore negative phase time [14]. A recent experiment by Robertson et al [16] on one asymmetric loop filter made of two different tubes has shown clearly the existence of such negative phase time and therefore negative group velocity.

In the last few years, the low-frequency band gap (called the locally resonant band gap) of a phononic crystal with small dimension has attracted much attention [17, 18]. 3D and 2D systems consisting of rubber-coated lead spheres and cylinders embedded in epoxy have shown the existence of such band gaps and their interest for blocking low-frequency sound [19, 20]. In addition to these gaps, great interest has been paid to the so-called Fano resonances that may be introduced in such gaps. Some analytical models have been proposed to explain the origin and the behavior of such resonances [18-20]. These resonances were first theoretically described by Fano [21] when he studied the inelastic autoionizing resonances in atoms. The asymmetry (Fano profile) was explained as a result of the interference between the discrete resonance and the smooth continuum background in which the former is embedded. The symmetric and asymmetric Fano line shapes have been extensively reported in the electronic transport in mesoscopic systems [22-24]. Mainly, the subject of these studies was to use the so-called AharonovBohm interferometric systems to show the conditions for the existence and the collapse of Fano resonances as functions of the applied current voltage and magnetic flux. These studies are also related to the investigation of the electronic states of quantum dots $[22,23]$ as well as to the understanding $[25,26]$ of the transmission phase jumps by $\pi$ between two adjacent resonances in relation to the experiments of Yacoby et al [27]. The analogy between scattering properties of electrons and phonons suggests that this type of feature can also appear in other vibrational systems [28].

The motivation behind the work presented in this paper is to introduce a design of a simple acoustic filter consisting of two slender side tubes, which play the role of resonators, grafted at two sites on an infinite slender tube (see figure 1). We show analytically and numerically that this simple structure can exhibit transmission gaps (their widths depend on the

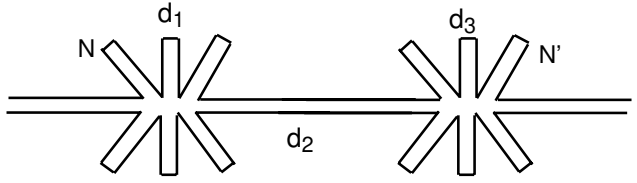

Figure 1. Schematic illustration of the one-dimensional slender tube waveguide of length $d_{2}$ with dangling resonators on both sides. The whole structure is inserted between two semi-infinite tubes. The lengths of the dangling resonators are $d_{1}$ and $d_{3}$ and their numbers are $N$ and $N^{\prime}$, respectively (here $N=N^{\prime}=6$ ). The boundary conditions at the end of the resonators are rigid.

number of dangling resonators) and Fano-like resonances. In particular, we show that the transmission amplitude through such a system can be written following the Fano-like shape around these resonances. In addition, we give an explicit expression of the Fano parameter [21] as well as the position and the width of the Fano resonances [21] as a function of the geometrical parameters of the system. It is worthwhile to notice that such stop bands and resonances could be observed experimentally by using simple slender tubes as in the recent experiments by Robertson et al [12,16].

This paper is organized as follows. In section 2, we give a brief review of the theoretical model used in this work as well as the analytical results of the structure depicted above. These results are necessary for an analytical understanding of the new phenomenon obtained for the structure proposed in this work. Section 3 is devoted to the transmission gaps and Fano resonances. The conclusions and some implications for future experiments are presented in section 4 .

\section{Method of theoretical and numerical calculation}

\subsection{Interface response theory of continuous media}

Our theoretical analysis is performed with the help of the interface response theory of continuous media, which allows calculation of the Green's function of any composite material. In what follows, we present the basic concept and the fundamental equations of this theory [35]. Let us consider any composite material contained in its space of definition $D$ and formed out of $N$ different homogeneous pieces located in their domains $D_{i}$. Each piece is bounded by an interface $M_{i}$, adjacent in general to $j(1 \leqslant j \leqslant J)$ other pieces through subinterface domains $M_{i j}$. The ensemble of all these interface spaces $M_{i}$ will be called the interface space $M$ of the composite material. The elements of the Green's function $g(D D)$ of any composite material can be obtained from [35]

$$
\begin{aligned}
& g(D D)=G(D D)-G(D M) G^{-1}(M M) G(M D) \\
& \quad+G(D M) G^{-1}(M M) g(M M) G^{-1}(M M) G(M D),
\end{aligned}
$$

where $G(D D)$ is the reference Green's function formed out of truncated pieces in $D_{i}$ of the bulk Green's functions of the infinite continuous media and $g(M M)$ the interface element of the Green's function of the composite system. The knowledge of the inverse of $g(M M)$ is sufficient to calculate the interface states of a composite system through the relation [35]

$$
\operatorname{det}\left[g^{-1}(M M)\right]=0 .
$$


Moreover, if $U(D)$ represents an eigenvector of the reference system, equation (1) enables the calculation of the eigenvectors $u(D)$ of the composite material and

$$
\begin{aligned}
& u(D)=U(D)-U(M) G^{-1}(M M) G(M D) \\
& \quad+U(M) G^{-1}(M M) g(M M) G^{-1}(M M) G(M D) .
\end{aligned}
$$

In equation (3), $U(D), U(M)$, and $u(D)$ are row vectors. Equation (3) provides a description of all the waves reflected and transmitted by the interfaces, as well as the reflection and transmission coefficients of the composite system. In this case, $U(D)$ is a bulk wave launched in one homogeneous piece of the composite material [17].

\subsection{Inverse surface Green's functions of the elementary constituents}

For the sake of simplicity, we embark on the simpler onedimensional system made of nonviscous fluid tubes. The calculations at hand simplify because the transverse speed of sound $v_{\mathrm{t}}$ is zero in nonviscous fluids. Nevertheless, the ordinary wave equation is inapplicable to inhomogeneous system. The correct wave equation, namely the equation of motion in the framework of the acoustic approximation, is

$$
\rho \frac{\partial^{2} \mathbf{u}}{\partial t^{2}}=\nabla\left[(\rho / a) v^{2} \nabla \cdot(a \mathbf{u})\right]
$$

where $\rho(\mathbf{r})$ is the mass density, $a(\mathbf{r})$ the cross section and $v(\mathbf{r})$ the longitudinal speed of sound. In the case of acoustic approximation, $\nabla \times(\rho \mathbf{u})=0$. Therefore, it is possible to define a scalar potential $\Phi(\mathbf{r}, t)$ such that $\rho \mathbf{u}=\nabla \Phi$. Then equation (4) may be cast in the form of a scalar equation

$$
\left[\frac{\rho}{a} v^{2}\right]^{-1} \frac{\partial^{2} \Phi}{\partial t^{2}}=\nabla \cdot\left[(\rho / a)^{-1} \nabla \Phi\right] .
$$

This is the starting point to be followed by the interface response theory [29].

In the following we consider a system made of homogeneous isotropic slender tubes $i$ full of the same fluid of mass density $\rho$ and velocity of sound $v_{0}$. Each tube is characterized by its impedance $Z_{i}=\rho v_{0} / a_{i}$, where $a_{i}$ is the cross section.

In the case of an infinite homogeneous one-dimensional slender tube along the $x$-axis, equation (5) becomes

$$
\left(\frac{\rho}{a}\right)^{-1}\left[\frac{\partial^{2} \Phi}{\partial x^{2}}-\alpha^{2}\right] \Phi(x)=0,
$$

where $\alpha^{2}=-\frac{\omega^{2}}{v_{0}^{2}}$ and $\omega$ is the angular frequency of the wave. Then, the corresponding Green's function is defined by [29]

$$
\left(\frac{\rho}{a}\right)^{-1}\left[\frac{\partial^{2} \Phi}{\partial x^{2}}-\alpha^{2}\right] G\left(x, x^{\prime}\right)=\delta\left(x-x^{\prime}\right),
$$

whose solution is given by

$$
G_{i}\left(x, x^{\prime}\right)=\frac{-\mathrm{j}}{2 \omega} Z_{i} \mathrm{e}^{-\alpha\left|x-x^{\prime}\right|}
$$

where $\alpha=-\mathrm{j} k, k=\omega / v_{0}$ and $\mathrm{j}=\sqrt{-1}$.
Before addressing the problem of the simple structure presented in this work (see figure 1), it is helpful to know the surface elements of its elementary constituents, namely, the Green function of a finite slender tube of length $d_{i}, i=1,2,3$, and of a semi-infinite tube. The finite slender tube of length $d_{2}$ is bounded by two free surfaces located at $x=0$ and $x=d_{2}$. These surface elements can be written in the form of a $(2 \times 2)$ matrix $g_{2}(M M)$, within the interface space $M=\left\{0,+d_{2}\right\}$. The inverse of this matrix takes the following form [29]:

$$
\left.g_{2}^{-1}(M M)=\begin{array}{cc}
-\frac{\omega C_{2}}{Z_{2} S_{2}} & \frac{\omega}{Z_{2} S_{2}} \\
\frac{\omega}{Z_{2} S_{2}} & -\frac{\omega C_{2}}{Z_{2} S_{2}}
\end{array}\right)
$$

$C_{2}=\cos \left(k d_{2}\right), S_{2}=\sin \left(k d_{2}\right)$. The inverse of the surface Green's functions of the dangling resonators grafted at the sites $\{0\}$ and $\left\{d_{2}\right\}$ with rigid boundary conditions at their ends is given by $g_{1}^{-1}(0,0)=-N \omega C_{1} / Z_{1} S_{1}$ and $g_{3}^{-1}\left(d_{2}, d_{2}\right)=$ $-N^{\prime} \omega C_{3} / Z_{3} S_{3}$, where $C_{i}=\cos \left(k d_{i}\right), S_{i}=\sin \left(k d_{i}\right), i=1,3$. $N$ and $N^{\prime}$ are the number of side branches on both sides of the finite slender tube of length $d_{2}$. The inverse of the surface Green's functions of the two semi-infinite tubes surrounding the whole structure is given by $g_{s}^{-1}(0,0)=g_{s}^{-1}\left(d_{2}, d_{2}\right)=$ $j \omega / Z_{s}$, where $Z_{s}=\rho v_{0} / a_{s}$. In what follows, we suppose that all the tubes have the same cross section (i.e. $a_{1}=$ $a_{2}=a_{3}=a_{s}=a$ ), or equivalently the same impedances (i.e. $Z_{1}=Z_{2}=Z_{3}=Z_{s}=Z=\rho v_{0} / a$ ). We report on results of calculated transmission coefficients and phase or phase time as a function of frequency. Using the Green's function method [29], the expression giving the inverse of the Green's function of the whole system given in the inset of figure 2(c) can be obtained from a linear superposition of the above inverse Green's functions of the constituent, namely

$$
g^{-1}(M M)=\frac{-\omega}{Z}\left(\begin{array}{cc}
\frac{C_{2}}{S_{2}}+\frac{N C_{1}}{S_{1}}-\mathrm{j} & -\frac{1}{S_{2}} \\
-\frac{1}{S_{2}} & \frac{C_{2}}{S_{2}}+\frac{N^{\prime} C_{3}}{S_{3}}-\mathrm{j}
\end{array}\right) .
$$

\subsection{Transmission coefficient}

Let us consider an incident wave $U(x)=\mathrm{e}^{-\mathrm{j} \alpha x}$ launched in the left semi-infinite waveguide (figure 1). With the help of equations (3), (8) and (10), one easily finds the transmission wave in the right semi-infinite waveguide (figure 1), namely [28, 29] $t=(2 \mathrm{j} \omega / Z) g\left(0, d_{2}\right)$, or equivalently

$$
t=\frac{2 S_{1} S_{3}}{\chi_{1}-\mathrm{j} \chi_{2}}
$$

where

$$
\chi_{1}=S_{2}\left(N S_{3} C_{1}+N^{\prime} S_{1} C_{3}\right)+2 C_{2} S_{1} S_{3}
$$

and

$$
\chi_{2}=-N C_{1}\left(S_{3} C_{2}+N^{\prime} S_{2} C_{3}\right)-N^{\prime} C_{2} C_{3} S_{1}+2 S_{1} S_{2} S_{3}
$$

From the expression of $t$ (equation (4)), one can deduce the transmission coefficient

$$
T=\frac{4 S_{1}^{2} S_{3}^{2}}{\chi_{1}^{2}+\chi_{2}^{2}}
$$



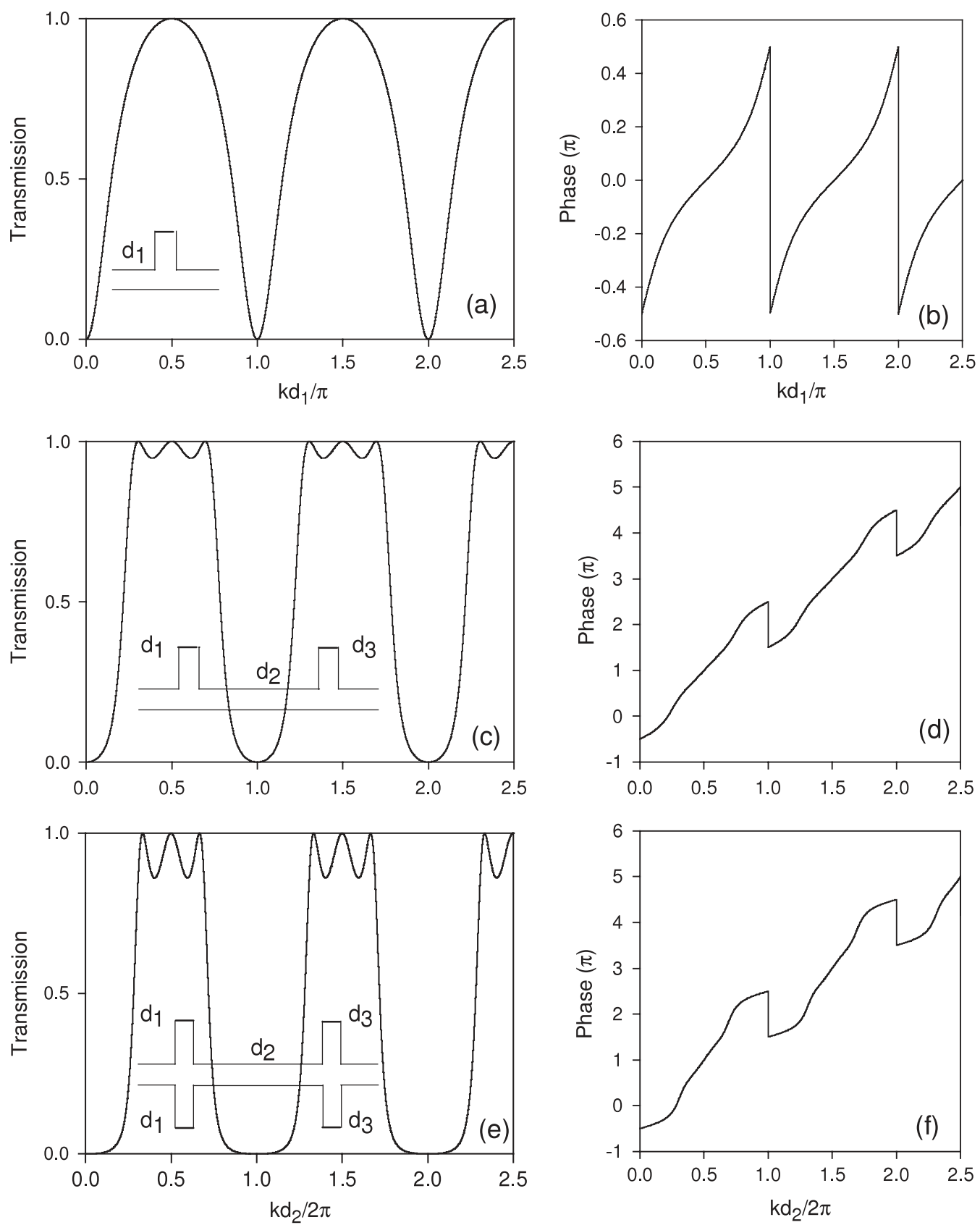

Figure 2. Left panel. (a) Transmission coefficient versus the reduced wavevector $k d_{1} / \pi$ for a structure (depicted in the inset) consisting of an infinite line with one grafted segment of length $d_{1}$. The one-dimensional tubes constituting the infinite line and the finite segment are assumed to be of the same cross section. The boundary conditions at the end of the stubs are rigid. (c) Transmission coefficient versus the reduced wavevector $k d_{2} / 2 \pi$ for the structure depicted in the inset with $d_{2}=2 d_{3}=2 d_{1}$. (e) The same as in (c) but for the structure depicted in the inset. Right panel. (b), (d), (f) The same as in the left panel but for the variation of the phase.

as well as the phase

$$
\varphi=\arctan \left(\chi_{2} / \chi_{1}\right)+\pi \Theta\left[S_{1} S_{3}\right]
$$

where $\Theta$ means the Heaviside function. From equations (4) and (7) one can notice that the transmission zeros are induced by the side branches (i.e. $S_{1}=0$ or $S_{3}=0$ ). When the expression $S_{1} S_{3}$ changes sign at some frequencies denoted by $\omega_{n}$, then the phase (equation (8)) exhibits a jump of $\pi$.

Another interesting quantity is the first derivative of $\varphi$ with respect to the frequency, which is related to the delay time taken by the phonons to traverse the structure. This quantity, called phase time, is defined by $[30,31]$

$$
\tau_{\varphi}=\frac{\mathrm{d} \varphi}{\mathrm{d} \omega}
$$

and can be written as

$$
\begin{aligned}
\tau_{\varphi}= & \frac{\mathrm{d}}{\mathrm{d} \omega} \arctan \left(\chi_{2} / \chi_{1}\right) \\
& +\pi \quad{ }_{n} \operatorname{sgn}\left[\frac{\mathrm{d}}{\mathrm{d} \omega}\left(S_{1} S_{3}\right)_{\omega=\omega_{n}}\right] \delta\left(\omega-\omega_{n}\right)
\end{aligned}
$$


where sgn means the sign function. Furthermore, the density of states (DOS) of the present composite system from which we have subtracted the DOS of the semi-infinite tubes is given by [31]

$$
\Delta n(\omega)=\frac{1}{\pi} \frac{\mathrm{d}}{\mathrm{d} \omega} \arctan \left(\chi_{2} / \chi_{1}\right) .
$$

Because of the second term in the right-hand side of equation (10), one can deduce that $\tau_{\varphi} \neq \pi \Delta n(\omega)$, as $\tau_{\varphi}$ (equation (10)) may exhibit $\delta$ functions at the transmission zeros that do not exist in the variation of the DOS (equation (11)). However, if the system does not exhibit transmission zeros, then $\Theta\left[S_{1} S_{3}\right]=0$ and $\tau_{\varphi}=\pi \Delta n(\omega)$. Let us mention that phase time and negative $\delta$ functions associated with the transmission zeros in asymmetric loop structures made of two different coaxial cables and slender tubes have been observed, respectively, in recent experiments by El Boudouti et al [32] and Robertson et al [16].

It should be pointed out again that the validity of our results is subject to the requirement $\sqrt{a_{i}} \ll d_{i}, \lambda$, i.e. the cross section of the slender tubes being negligible compared to their length and to the propagation wavelength $\lambda$. The assumption of monomode propagation is then satisfied. It is worthwhile to notice that this model has been applied successfully to reproduce experimental measurements on band gaps, defect modes and group velocities in structured acoustic waveguides $[7,8,12,16]$.

\section{Transmission gaps and Fano resonances}

Before addressing the problem of the whole structure described above (figure 1), let us first recall briefly the results of a particular case necessary for the understanding of wave propagation in the structures shown in figure 1; namely, if $d_{2}=0, N=1$ and $N^{\prime}=0$, we obtain the transmission function of a simple structure consisting of one resonator grafted on an infinite guide (see the inset of figure 2(a)): $t=S_{1} /\left(S_{1}+\mathrm{j} C_{1} / 2\right)$. This expression enables us to deduce the transmission coefficient $T=|t|^{2}=4 S_{1}^{2} /\left(4 S_{1}^{2}+C_{1}^{2}\right)$ and the phase $\varphi=\pi \Theta\left(S_{1}\right)-\arctan \left(C_{1} / 2 S_{1}\right)$.

We can see that the transmission coefficient is equal to zero when $k d_{1}=l^{\prime} \pi$, where $l^{\prime}$ is a positive integer. The variation of $T$ versus the reduced wavevector $k d_{1}$ (or equivalently the reduced frequency $\left.\omega d_{1} / v_{0}\right)$ is reported in figure 2(a). $T$ is equal to zero for $k d_{1}$ a multiple of $\pi$ and reaches its maximum value of unity for $k d_{1}$ an odd multiple of $\pi / 2$. The frequencies of the transmission zeros given by $f_{\mathrm{g}}$ such as $f_{\mathrm{g}}=l^{\prime} v_{0} / 2 d_{1}$ correspond to the eigenmodes of the grafted finite branch. This grafted branch behaves as a resonator and this simple composite system filters out the modes $f_{\mathrm{g}}$. This phenomenon is related to the resonances associated with the finite additional path offered to the acoustic wave propagation. The variation of the phase versus $k d_{1}$ (figure 2(b)) shows an abrupt change of $\pi$ at the transmission zeros and therefore the corresponding phase time is different from the DOS as mentioned above.

For the structure shown in the inset of figure 2(c), equation (4) clearly shows that the transmission zeros are due only to the dangling resonators (i.e. when $S_{1}=0$ or $S_{3}=0$ ).
Figure 2(c) gives the transmission coefficient in the presence of two identical dangling resonators (i.e. $N=N^{\prime}=1$ and $d_{1}=$ $d_{3}=0.5 d_{2}$ ). One can notice that the transmission coefficient presents well defined dips induced by the grafted branches. This dip transforms into a large transmission gap when the number of branches increases, as illustrated in figure 2(e) for $N=N^{\prime}=2$. It is worthwhile to mention that, because of the existence of two resonators in figure 2(c), one can expect two phase drops of $\pi$ (i.e. $2 \pi$ ) at the transmission zeros given by $S_{1}=S_{3}=0$ (i.e. $k d_{2} / 2 \pi=1,2, \ldots$ ). However, one can see in figure $2(\mathrm{~d})$ that the phase presents only a phase drop of $\pi$. This is due to the existence of a resonant state with zero width at these values of $k d_{2} / 2 \pi$, which induces a phase jump of $+\pi$; these resonances collapse when $d_{1}=d_{3}$ is taken exactly equal to $0.5 d_{2}$. These resonances are called ghost Fano resonances in $[33,34]$. To enlarge these resonances, we have to take $d_{1}$ and $d_{3}$ slightly different from $0.5 d_{2}$. Indeed, at $k d_{2} / 2 \pi=1,2, \ldots$ the expression of the transmission function (equation (4)) becomes

$$
t=\frac{2 S_{1} S_{3}}{2 S_{1} S_{3}+\mathrm{j} \sin \left[k\left(d_{1}+d_{3}\right)\right]} .
$$

So, if $k\left(d_{1}+d_{3}\right)=m \pi$ but $k d_{1} \neq m_{1} \pi$ and $k d_{2} \neq m_{2} \pi$ ( $m, m_{1}$ and $m_{2}$ are integers), one obtains a resonance that reaches unity (i.e. $t=1$ ). An example corresponding to this situation is given in figure 3(a), where $d_{1}=0.46 d_{2}$ and $d_{3}=$ $0.54 d_{2}$ (with $d_{1}+d_{3}=d_{2}$ ). One can notice that the resonance at $k d_{2} / 2 \pi=1$ is squeezed between two zeros (indicated by solid circles on the abscissa of figure 3(a)) induced by the dangling resonators, as also illustrated in the curve of the variation of the phase (figure 3(b)). The width of this resonance increases as $d_{1}$ and $d_{3}$ deviate from $0.5 d_{2}$ (see below). In the particular case where $k d_{1}=m_{1} \pi$ and $k d_{2}=m_{2} \pi$, the numerator and denominator of $t$ (equation (12)) vanishes altogether. In this case, the resonance as well as the two zeros induced by the resonators fall at the same position, so the resonance collapses, the transmission coefficient vanishes and the phase drops by $\pi$ as shown in figure 2(d).

The resonance in figure 3(a) shows the same characteristics as a Fano resonance but with two zeros of transmission around the resonance instead of one as is usually the case [21, 22]. Indeed, one can obtain an approximate analytical expression for the transmission function (equation (4)) in the vicinity of the resonance. A Taylor expansion around $k d_{2}=2 \pi$ (i.e. $k d_{2}=2 \pi+\varepsilon$ with $\varepsilon / 2 \pi \ll 1$ ) enables us to obtain

$$
t=\frac{\zeta \zeta^{\prime}}{2 \varepsilon^{2}+\zeta \zeta^{\prime}+\mathrm{j} \varepsilon\left(4-\zeta \zeta^{\prime} / 2\right)}
$$

where $\zeta=2 \Delta+\varepsilon(1+\Delta / \pi), \zeta^{\prime}=-2 \Delta+\varepsilon(1-\Delta / \pi)$ and $\Delta$ is the detuning of $d_{1}$ and $d_{3}$ from $0.5 d_{2}$ (i.e. $\Delta=$ $\left.2 \pi\left(0.5-d_{1} / d_{2}\right)=2 \pi\left(-0.5+d_{3} / d_{2}\right)\right)$.

From equation (12), one can show that the transmission coefficient $T$ can be written (following the Fano line shape [21]) in the form

$$
T=A \frac{\left(\varepsilon+q_{1} \Gamma\right)^{2}\left(\varepsilon-q_{2} \Gamma\right)^{2}}{\varepsilon^{2}+\Gamma^{2}},
$$



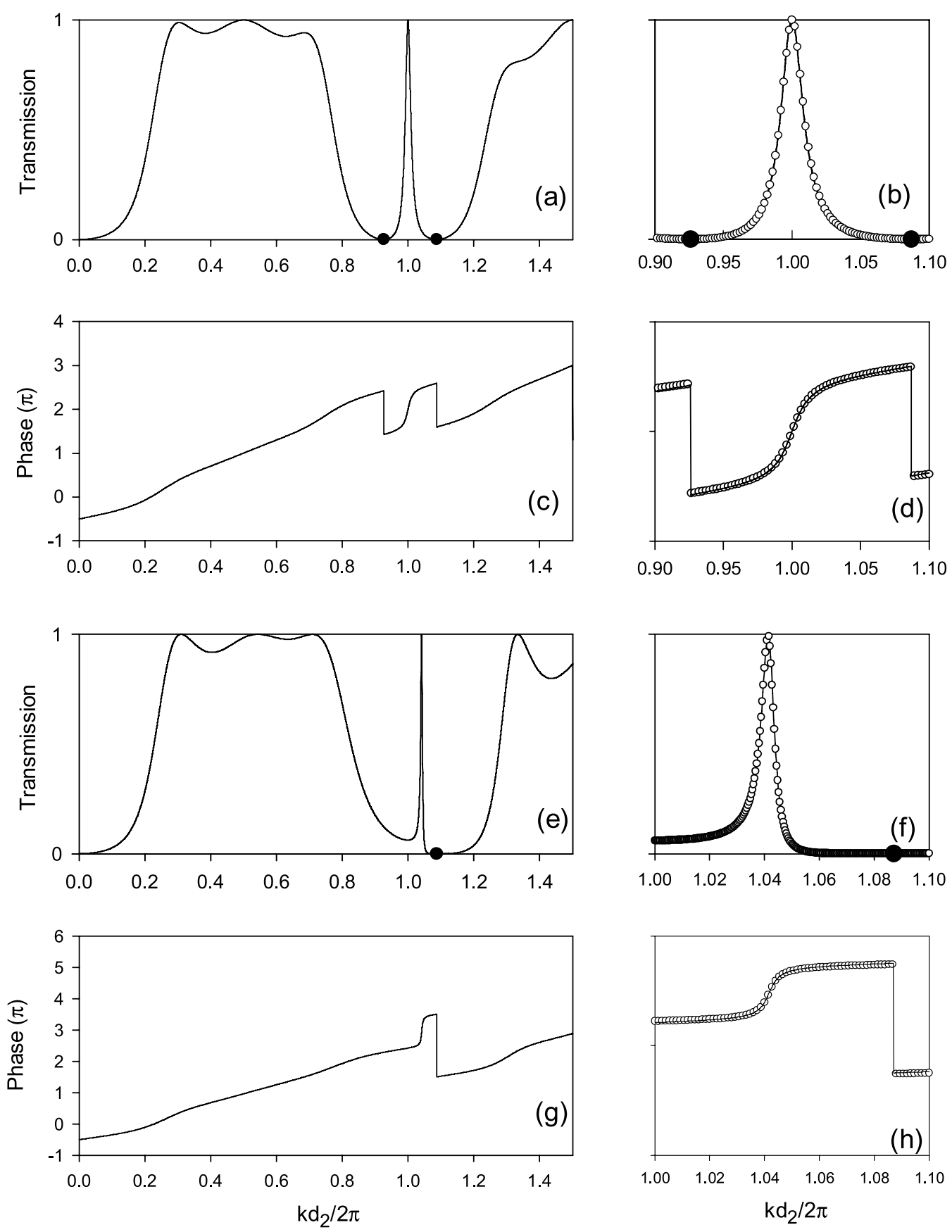

Figure 3. (a) The same as in figure 2(a), but the lengths of the resonators are taken such that $d_{1}=0.46 d_{2}, d_{3}=0.54 d_{2}$ and $N=N^{\prime}=1$. Solid circles on the abscissa indicate the positions of the transmission zeros induced by the dangling resonators on both sides of the resonance. (c) The same as in (a) but for the variation of the phase. (b), (d) The approximate results (open circles) around the resonance. (e) The same as in (a) but the resonators are taken to be of identical lengths $d_{1}=d_{3}=0.46 d_{2}$. (g) The same as in (c) but for the variation of the phase. (f), (h) The approximate results (open circles) around the resonance.

where $A=\left(1-\Delta^{2} / \pi^{2}\right)^{2} /\left(4+2 \Delta^{2}\right)^{2} . \Gamma=2 \Delta^{2} /\left(2+\Delta^{2}\right)$ characterizes the width of the resonance falling at $\varepsilon=0$. $q_{1}=\left(2+\Delta^{2}\right) / \Delta(1+\Delta / \pi)$ and $q_{2}=\left(2+\Delta^{2}\right) / \Delta(1-$ $\Delta / \pi)$ are the coupling parameters; they give qualitatively the interference between the bound states and the propagating continuum states [21-23]. One can notice that when increasing $\Delta, \Gamma$ increases and $q$ decreases. The results of the approximate expression (equation (14)) are shown in figure 3(b) by open circles. These results are in accordance with the exact ones (solid lines) and clearly show that the resonance is of Fano type with $q_{1} \simeq 7.6, q_{2} \simeq 8.9$ and width $2 \Gamma \simeq 0.12$. The commonly studied Fano resonances are asymmetric because of the presence of only one transmission zero near the resonance (see below). In addition, in the electronic counterpart studies, a perturbation is often introduced to the system in order to create the resonance state [21-24]. However, the above calculation shows that, without introducing any perturbation in the structure, one can find a well defined symmetric 
Fano resonance with a width $2 \Gamma$ and coupling parameters $q_{1}$ and $q_{2}$ that can be adjusted by tailoring the lengths of the resonators (i.e. $\Delta$ ). Equation (13) enables us also to deduce an approximate expression for the phase as

$$
\varphi=\pi \Theta(\zeta)+\pi \Theta\left(\zeta^{\prime}\right)-\arctan \left[\varepsilon\left(4-\zeta \zeta^{\prime} / 2\right) /\left(2 \varepsilon^{2}+\zeta \zeta^{\prime}\right)\right]
$$

This function is plotted by open circles in figure 3(d) and clearly shows two abrupt phase changes of $\pi$ at $\zeta=0$ and $\zeta^{\prime}=0$ (i.e. $\varepsilon_{1}=-q_{1} \Gamma$ and $\varepsilon_{2}=q_{2} \Gamma$ ) in accordance with the exact results (solid line).

One can also create an asymmetric Fano resonance by adjusting the transmission zeros on only one side of the resonance; this can be obtained by considering a structure where the resonators are supposed to be identical with lengths slightly different from $0.5 d_{2}$. This is shown in figure 3(e) for $d_{1}=d_{3}=0.46 d_{2}$ and $N=N^{\prime}=1$. Indeed, an analytical Taylor expansion around $k d_{2}=2 \pi$ enables us to write the transmission function (equation (14)) as

$$
t=\frac{2 \xi^{2}}{(\xi+\mathrm{j})(\varepsilon+2 \xi-\mathrm{j} \varepsilon \xi)}
$$

where $\xi=\Delta+\varepsilon(1+\Delta / \pi) / 2$ and $\Delta$ is the detuning of the lengths of the two resonators from $0.5 d_{2}$ (i.e., $\Delta=2 \pi\left(d_{1} / d_{2}-\right.$ $0.5)$ ).

From the expression of $t$ (equation (16)), one can deduce the following Fano line shape transmission coefficient:

$$
\begin{aligned}
T= & B \frac{1}{1+\xi^{2}} \frac{\left(\varepsilon-\varepsilon_{\mathrm{R}}+q \Gamma\right)^{4}}{\left(\varepsilon-\varepsilon_{\mathrm{R}}\right)^{2}+\Gamma^{2}} \\
& \simeq B \frac{\left(\varepsilon-\varepsilon_{\mathrm{R}}+q \Gamma\right)^{4}}{\left(\varepsilon-\varepsilon_{\mathrm{R}}\right)^{2}+\Gamma^{2}}
\end{aligned}
$$

where $B=(1+\Delta / \pi)^{4} / 16(1+\Delta / 2 \pi)^{2}$.

$\Gamma=\Delta^{2}(1-\Delta / 2 \pi) / 4(1+\Delta / 2 \pi)^{2}$ and $\varepsilon_{\mathrm{R}}=-\Delta /(1+$ $\Delta / 2 \pi)$ characterize the width and the shift of the resonance respectively, whereas $q=4(1+\Delta / 2 \pi) / \Delta(1+\Delta / \pi)(1-$ $\Delta / 2 \pi)$ is the Fano parameter. One can notice that the resonance shifts slightly from $k d_{2}=2 \pi$ and its width is small as compared to the preceding case; this is in accordance with the numerical results of figures 3(a) and (e). Also $q$ increases when $\Delta$ decreases and tends to infinity when $\Delta$ vanishes. In this case the resonance falls at $\varepsilon_{\mathrm{R}}=0$ and its width $2 \Gamma$ reduces to zero (figure $2(\mathrm{c})$ ), as expected. The results of the approximate expression (equation (17)) are sketched (open circles) in figure 3 (f) for $\Delta=2 \pi\left(d_{1} / d_{2}-0.5\right)=-0.08 \pi$ (i.e. $d_{1} / d_{2}=0.46$ ). These results are in accordance with the exact ones (solid lines) and clearly show that the resonance is of Fano type with $q \simeq 16$ and width $2 \Gamma \simeq 0.035$.

Concerning the evolution of the phase of the phonons in this structure, one can notice from equation (4) that the numerator of the transmission function $t$ vanishes when $S_{1}=$ $S_{3}=0$ (or equivalently $\xi=0$ in the approximate result (equation (16)) at $k d_{2} / 2 \pi=d_{2} / 2 d_{1}=1.086$ indicated by a filled circle on the abscissa axis of figure 3(f). The transmission zeros induced by the two identical resonators fall at the same frequency; therefore, the phase (figures $3(\mathrm{~g})$ and $(\mathrm{h})$ ) shows a phase drop of $2 \pi$ at these frequencies. Indeed, as the phase is defined modulo $2 \pi$, the $2 \pi$ phase change cannot be observed if the absorption is neglected in the system. The absorption can be considered by adding for example a small imaginary part $\varepsilon$ to the frequency $\omega$ with $\varepsilon \approx 0$, then the abrupt phase drop can be observed. The phase drops give rise to negative delta peaks in the transmission phase time as illustrated in figures 4(c) and $(\mathrm{g})$ as well as in the corresponding approximative results shown by open circles in figures $4(\mathrm{~d})$ and (h). A recent similar work has been performed by Rotter and Sadreev [35] on two single quantum dots coupled by a wire of finite length and neglecting the absorption. They found that at the transmission zeros the phases jump by mostly (but not always) $\pi$. However, our results show that the phase may jump by even $2 \pi$ if the absorption is taken into account.

The small absorption in the tubes can also considerably affect the amplitude of the Fano resonances, which becomes less than unity as shown in figures 4(a), (b), (e) and (f). It is worthwhile to notice that recent experiments by Robertson et al [16] on an asymmetric loop made of slender tubes have shown the existence of negative phase times and therefore negative group velocities around the transmission zeros in analogy with our experiments on photonic crystals made of coaxial cables [32]. These results show, in accordance with section 2, that the phase time is different from the density of states by the occurrence of additional delta peaks [14].

The Green's function approach enables one also to deduce the local density of states (LDOS). The details of these calculations are given in [28]. The LDOS reflects the behavior of the square modulus of the pressure field inside the structure. An analysis of the LDOS as a function of the space position (figure 5) clearly shows that the Fano resonances in figures 3(a) and (e) are confined inside the slender tube and the resonators (see figures 5(a) and (b) respectively). In particular, the pressure is maximum in the middle of the finite tubes and vanishes at their extremities. Therefore, these resonances could be classified as local resonances.

As mentioned above, through our analysis we use the one-dimensional model for the acoustic waveguide network where the cross section of the slender tubes is negligible as compared to their length and to the propagation wavelength $\lambda$. However, if the cross section of the slender tubes is of the same order of magnitude as compared to their length and to the propagation wavelength $\lambda$, then a two-dimensional model is necessary [10, 36, 37]. Indeed, it was shown recently $[36,37]$ that the transmission zeros induced by the stubs in two-dimensional phononic crystals strongly depend on the number of stubs grafted at each site as well as on the distance between the stubs. Similar results to those presented in this work have been observed in quantum waveguide nanostructures [38]. In particular, when the cross sections of the wires are of nonzero width, the multimode effect and the matching of transverse modes have been considered [39]. In addition to the quantization of the conductance which is due to the lateral confinement, It has been shown that the existence of crosses and bends in the structure may also result in bound states associated with the cross sections of these contact points [40-43]. One can expect the same phenomena in acoustic waveguides due to the analogy between electronic and acoustic excitations in waveguides. Therefore, in such 

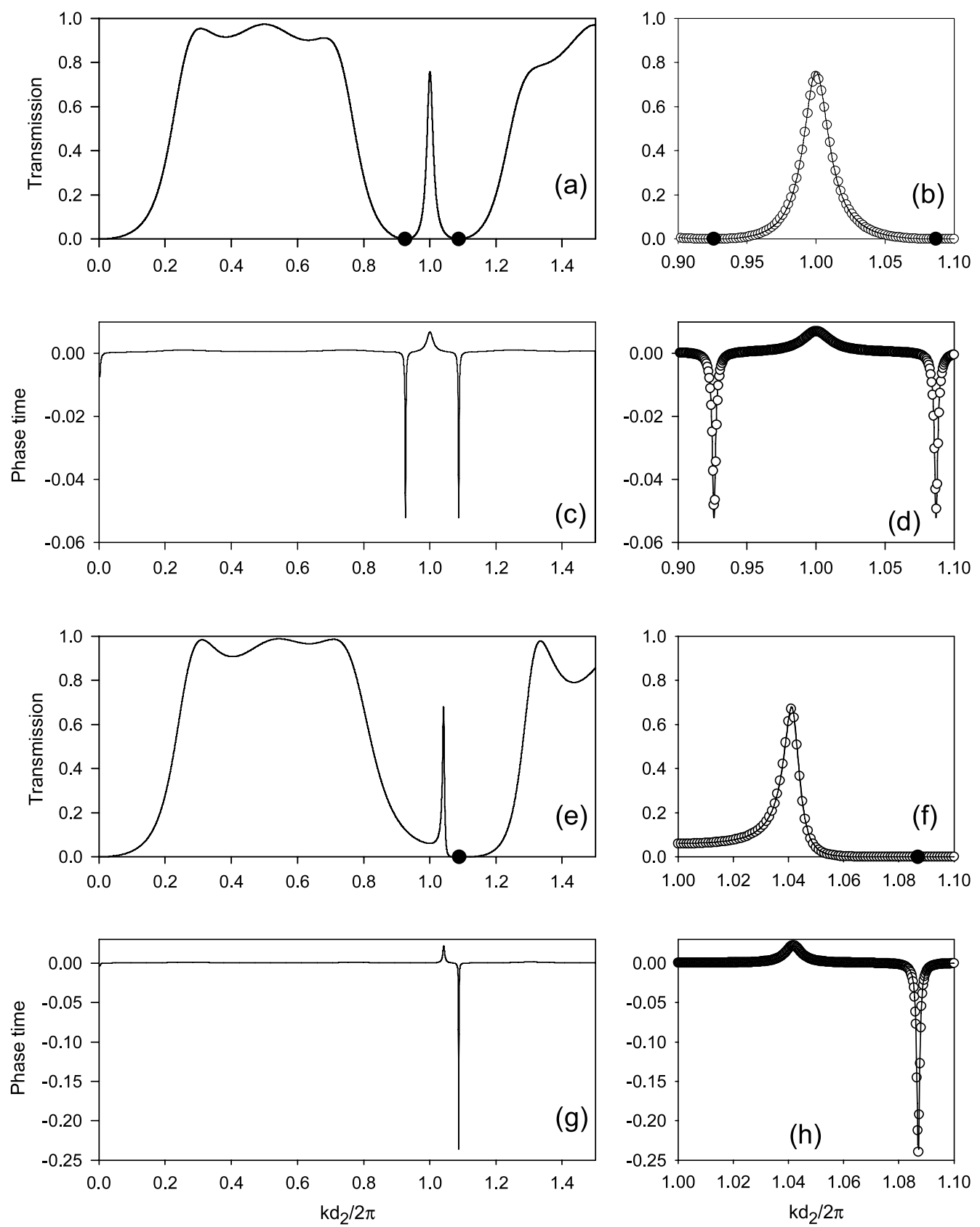

Figure 4. (a), (b), (e), (f) The same as in figures 3(a), (b), (e), (f) but here the absorption is taken into account by adding a small imaginary part to the frequency. The solid circles indicate the positions of the transmission zeros. (c), (d), (g), (h) The same as in figures 3(c), (d), (g), (h) but for the phase time.

geometries, the position and the width of the transmission gaps as well as the position and intensity of the Fano resonances in the acoustic counterpart systems could be affected.

\section{Summary and conclusion}

In summary, we have clearly demonstrated that a simple geometry of a slender tube with dangling side resonators on both sides can pave the way to the obtention of gaps in the sound propagation. The existence of the stop bands in the spectrum is attributed to the zero of transmission associated with the dangling resonators. The width of the transmission gaps depends on the number of the side resonators grafted on both sides of the slender tube. Besides the transmission gaps, we have shown the existence of asymmetric and symmetric Fano resonances that may lie near the vicinity of a transmission zero or be squeezed between two transmission zeros. These resonances are obtained by tailoring the lengths of the different branches constituting the structure. A study of the phase of the transmission function enables us to deduce several properties of the wave propagation through such structures as the phase times and therefore the density of states. For the system studied here, the phase time calculation is different from the density of states. The resonant modes give rise to well defined peaks in the phase time. 

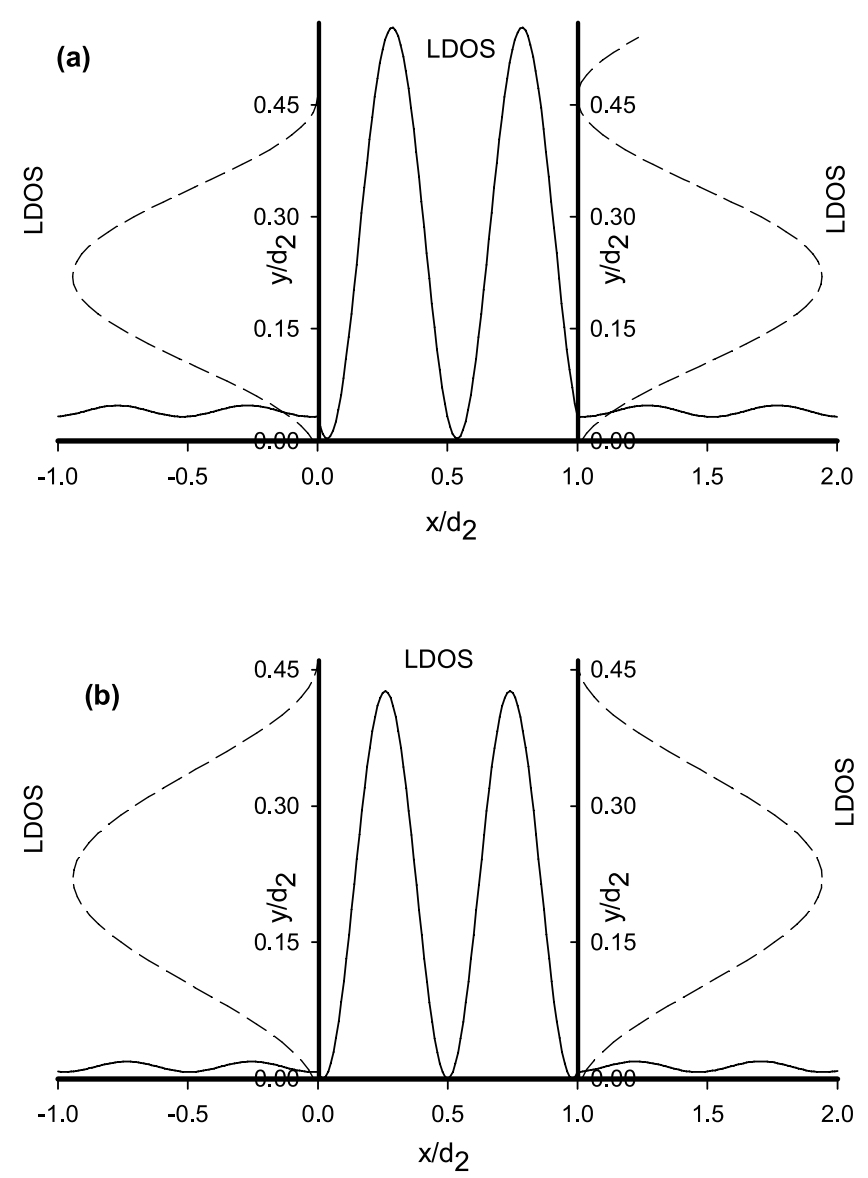

Figure 5. The local density of states (LDOS) (in arbitrary units) as a function of the space positions $x / d_{2}$ and $y / d_{2}$ along the horizontal waveguide (full curve) and vertical waveguide (dashed curves) respectively. (a) The LDOS corresponds to the Fano resonance in figure 3(a); (b) the same as in (a) but for the resonance in figure 3(e).

The advantage of the simple acoustic waveguide model presented in this work consists in finding simple analytical expressions that enable us to discuss the existence of Fano resonances as well as the effect of the different tube lengths in tailoring these resonances without incorporating a defect in the structure as is usually the case in the electronic counterpart studies [21-24].

We believe that this paper brings a new piece of work in the field of acoustic wave transport in 1D waveguide structures and we hope that it will stimulate an experimental observation $[12,16]$ of the transmission gaps and Fano resonances exhibited by the simple acoustic waveguide described in this work.

\section{Acknowledgments}

E H El Boudouti and $\mathrm{H}$ Al-Wahsh gratefully acknowledge the hospitality of the Institut d'Electronique, de Microélectronique et de Nanotechnologie (IEMN), UMR CNRS 8520 and UFR de Physique, Université de Lille 1. This work was supported by 'le Fond Européen de Développement Régional' (FEDER), INTERREG III France-Wallonie-Flandre (PREMIO) and 'le Conseil Régional Nord-Pas de Calais'.

\section{References}

[1] Kushwaha M S, Halevi P, Dobrzynski L and Djafari-Rouhani B 1993 Phys. Rev. Lett. 712022

Sigalas M and Economou E N 1993 Solid State Commun. 86141

Sainidou R, Stefanou N, Psarobas I E and Modinos A 2002 Phys. Rev. B. 66024303

[2] Montero de Espinosa F R, Jimenez E and Torres M 1998 Phys. Rev. Lett. 801208

Vasseur J O, Deymier P A, Frantziskonis G, Hong G and Djafari-Rouhani B 1998 J. Phys.: Condens. Matter 106051

[3] Sigalas M, Kushwaha M S, Economou E N, Kafesaki M, Psarobas I E and Steurer W 2005 Z. Kristallogr. 220765

[4] Narayanamurti V 1981 Science 213717

[5] Bria D and Djafari-Rouhani B 2002 Phys. Rev. E 66056609 and references therein

[6] Manzanarez-Martinez B, Sanchez-Dehesa J, Hakansson A, Cervera F and Ramos-Mendieta F 2004 Appl. Phys. Lett. 85154

[7] Bradley C E 1994 J. Acoust. Soc. Am. 961844

Bradley C E 1994 J. Acoust. Soc. Am. 961854

Bradley C E 1995 J. Acoust. Soc. Am. 188717

Bradley C E 1996 J. Acoust. Soc. Am. 100304

[8] Munday J N, Bennett C B and Robertson W M 2002 J. Acoust. Soc. Am. 1121353

Robertson W M, Baker C and Bennett C B 2004 Am. J. Phys. 72255

[9] Kushwaha M S, Akjouj A, Djafari-Rouhani B, Dobrzynski L and Vasseur J O 1998 Solid State Commun. 106659

[10] Wang X F, Kushwaha M S and Vasilopoulos P 2001 Phys. Rev. B 65035107

[11] Carr C and Yu R 2002 Am. J. Phys. 701154

[12] Robertson W M, Ash J and McGaugh J M 2002 Am. J. Phys. 70689

[13] Akjouj A, Al-Wahsh H, Sylla B, Djafari-Rouhani B and Dobrzynski L 2004 J. Phys.: Condens. Matter 1637

[14] Aynaou H, El Boudouti E H, Djafari-Rouhani B, Akjouj A and Velasco V R 2005 J. Phys.: Condens. Matter 174245

[15] Kinga P D C and Cox T J 2007 J. Appl. Phys. 102014902

[16] Robertson W M, Pappafotis J and Flannigan P 2007 Appl. Phys. Lett. 90014102

[17] Liu Z, Zhang X, Mao Y, Zhu Y Y, Yang Z, Chan C T and Sheng P 2000 Science 2891734

Yang S, Page J H, Liu Z, Cowan M L, Chan C T and Sheng P 2002 Phys. Rev. Lett. 88104301

[18] Goffaux C, Sánchez-Dehesa J, Yeyati A L, Lambin P, Khelif A, Vasseur J O and Djafari-Rouhani B 2002 Phys. Rev. Lett. 88225502

Goffaux C and Sánchez-Dehesa J 2003 Phys. Rev. B 67144301

[19] Liu Z, Chan C T and Sheng P 2005 Phys. Rev. B 71014103

[20] Kosevich Y A, Goffaux C and Sánchez-Dehesa J 2006 Phys. Rev. B 74012301

[21] Fano U 1961 Phys. Rev. 1241866

[22] Goldhaber-Gordon D, Shtrikman H, Mahalu D, Abush-Magder D, Meirav U and Kastner M A 1988 Nature 391156

Gores J, Goldhaber-Gordon D, Heemeyer S, Kastner M A, Shtrikman H, Mahalu D and Meirav U 2000 Phys. Rev. B 622188

Ji Y, Heiblum M and Shtrikman H 2002 Phys. Rev. Lett. 88076601

[23] Kobayashi K, Aikawa H, Katsumoto S and Iye Y 2002 Phys. Rev. Lett. 88256806

Kim J, Kim J R, Lee J-O, Park J W, So H M, Kim N, Kang K, Yoo K-H and Kim J-J 2003 Phys. Rev. Lett. 90166403

Babic B and Schönenberger C 2004 Phys. Rev. B 70195408 
[24] Ladron de Guevara M L, Claro F and Orellana P A 2003 Phys. Rev. B 67195335

Voo K K and Chu C S 2005 Phys. Rev. B 72165307

Hackenbroich G and Weidenmuller H A 1996 Phys. Rev. Lett. 76110

Joe Y S, Satanin A M and Klimeck G 2005 Phys. Rev. B 72115310

[25] Wu J, Gu B L, Chen H, Duan W and Kawazoe Y 1998 Phys. Rev. Lett. 801952

Taniguchi T and Buttiker M 1999 Phys. Rev. B 6013814

Lee H W 1999 Phys. Rev. Lett. 822358

[26] Al-Wahsh H, El Boudouti E H, Djafari-Rouhani B, Akjouj A and Dobrzynski L 2007 Phys. Rev. B 75125313

[27] Yacoby A, Heiblum M, Mahalu D and Shtrikman H 1995 Phys. Rev. Lett. 744047

Schuster R, Buks E, Heiblum M, Mahalu D, Umansky V and Shtrikman H 1997 Nature 385417

[28] Vasseur J O, Akjouj A, Dobrzynski L, Djafari-Rouhani B and El Boudouti E H 2004 Surf. Sci. Rep. 541

[29] Dobrzynski L 1990 Surf. Sci. Rep. 11139

[30] Büttiker M and Landauer R 1982 Phys. Rev. Lett. 491739 Büttiker M 1983 Phys. Rev. B 276178

Hauge E H and Stovneng J A 1989 Rev. Mod. Phys. 61917

[31] Lahlaouti M L H, Akjouj A, Djafari-Rouhani B, Dobrzynski L, Hammouchi M, El Boudouti E H, Nougaoui A and Kharbouch B 2001 Phys. Rev. B 6335312

[32] El Boudouti E H, Fettouhi N, Akjouj A, Djafari-Rouhani B, Mir A, Vasseur J, Dobrzynski L and Zammouri J 2004 J. Appl. Phys. 951102
[33] Ladrón de Guevara M L, Claro F and Orellana Pedro A 2003 Phys. Rev. B 67195335

[34] Barkay H, Narevicius E and Moiseyev N 2003 Phys. Rev. B 67045322

[35] Rotter I and Sadreev A F 2005 Phys. Rev. E 71046204

[36] Benchabane S, Khelik A, Choujaa A, Djafari-Rouhani B and Laude V 2005 Europhys. Lett. 71570

[37] Noual A 2006 MS Thesis University of Lille, p 1

[38] Levy L P, Dolan G, Dunsmuir J and Bouchiat H 1990 Phys. Rev. Lett. 642074

Deo P S and Jayannavar A M 1990 Mod. Phys. Lett. B 72074

Deo P S and Jayannavar A M 1994 Phys. Rev. B 5011629

[39] Van Wees B J, van Houten H, Beenakker C W J, Williamson J G, Kouwenhoven L P, Van der Marel D and Foxon C T 1988 Phys. Rev. Lett. 60848

Debray P, Raichev O E, Vasilopoulos P, Rahman M, Perrin R and Mitchell W C 2000 Phys. Rev. B 6110950

[40] Berggren K F and liJi Z- 1991 Phys. Rev. B 434760

[41] Carini J P, Londergan J T and Murdock D P 1997 Phys. Rev. B 559852

Carini J P, Londergan J T, Murdock D P, Trinkle D and Yung C S 1997 Phys. Rev. B 559842

[42] Avishai Y, Bessis D, Giraud B G and Mantica G 1991 Phys. Rev. B 448028

[43] For a review, see Londergan J T, Carini J P and Murdock D P 1999 Binding and Scattering in Two-Dimensional Systems: Application to Quantum Wires, Waveguides and Photonic Crystals (LNP vol m60) (Berlin: Springer) 